\title{
Fuzzy Optimization Research of Product Design Improvement Based on C \#
}

\author{
Hui Kang ${ }^{1}$, Yong Wang ${ }^{2}$ and Shuo Zhou ${ }^{3}$ \\ North China Electric Power University, Baoding 071000, China
}

wy13191687828@163.com

Keywords: product design improvements, fuzzy comprehensive evaluation, C \#.

\begin{abstract}
The purpose of the research is using fuzzy comprehensive evaluation rule of fuzzy optimization method to assess the weights of the product indicators and then improve the product design according to the optimization results. First, we selected an appropriate fuzzy optimization method and established a mathematical model of fuzzy optimization according to the actual requirements of the design. Then we obtain the optimal solution of fuzzy optimal design by computer and improved the indicators of the product. Finally we got a new design. The fuzzy optimization design can improve the design of product. C \# plays a significant role due to the powerful ability of processing large amounts of data.
\end{abstract}

\section{Introduction}

Improving the design of products can reduce the costs of manufacture, improve the performance and longevity and enhance the competitiveness of the products. An excellent product design can reduce the negative impact on the environment by saving energy and making the recycle and reuse of resources more easily. Furthermore, by researching fuzzy optimization in product design, a designer can grasp some specific application methods in common modern design. In order to open up new consumer markets, it is also necessarily to a designer to give the product design a new design method or use a new method to give the product a new feature with an innovative spirit. It will enhance an enterprise or even a country's competitiveness in the process of economic globalization.

\section{$1.1 \mathrm{C \#}$}

$\mathrm{C \#}$ is a safe, stable and simple object-oriented programming out language derived from $\mathrm{C}$ and $\mathrm{C}++$. C\# combines the simple visualization of $\mathrm{VB}$ and the high operating efficiency of $\mathrm{C}++$. It became the preferred language in NET developed with its powerful operation ability, elegant syntax styles, innovative language features and ease support of component-oriented programming.

\subsection{Product Design Improvement}

Product design improvement is a re-development design to optimize, enrich and improve the existing products. Product design improvement is an important part of the innovative design. It is also an important research topic for industrial designer. Product design improvement should be modified from existing products. Designer should have an objective and comprehensive analysis of the product's advantages and disadvantages. The use environments and conditions of products in the past, present and future should also be considered.

\subsection{Fuzzy Optimization Design}

Due to the widespread ambiguity in the transition of things' differences, it will inevitably encounter a large number of fuzzy conceptions in the process of product design. Sometimes a designer need to describe which product has a more reasonable, comfortable and beautiful appearance or a better quality in work. Fuzzy optimization design includes two parts, building a mathematical model and using computer programs to solve the model. How to abstract a mathematical model correctly from the practical problem is one of the key points of projects fuzzy optimization design. It is also the priorities of engineering staff in fuzzy optimization design. Liking other conventional optimization design, the design variables, objective function and constraints are the three elements of fuzzy optimization design mathematical model. 
Design variables:

$$
X=\left(x_{1}, x_{2}, \ldots, x_{n}\right)^{T}
$$

Objective function:

$$
\operatorname{minf}(X)
$$

Constraints:

$$
\mathrm{g}_{\mathrm{j}}(X) \subset G_{\mathrm{j}} \quad \mathrm{j}=1,2, \ldots, \quad J
$$

\section{Product Design Improvement with Fuzzy Optimization Design}

A great medical device manufacturer need a new design of fluid infusion instrument which could heating and warning, so they analyzed the hospitals' opinions on the existing instrument (Figure 2.1) to determine how to make improvements. Through the survey they found that the product performance, product shape and product color are heavily considered factors of a hospital, so they designed the improved product (Figure 2.2). Before production, the need to judge whether the instrument is the best, so they used fuzzy optimization design to analyze it. They judged the product by its performance, shape and color. Hospitals' opinions on the instrument can be divided into very reasonable, reasonable, less reasonable and unreasonable.

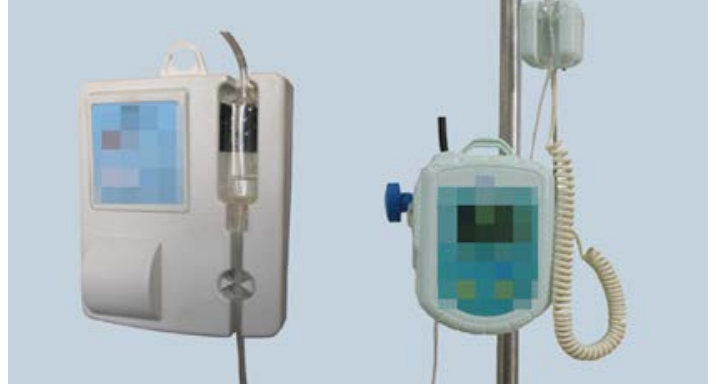

Figure 2.1 Existing instruments

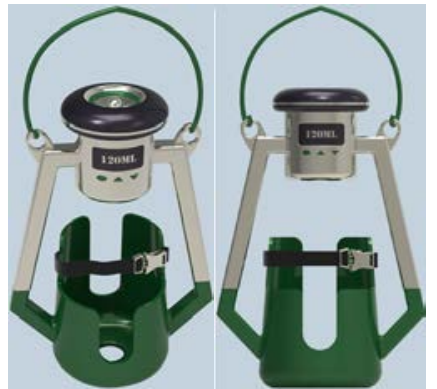

Figure 2.2 Improved instruments

They collected some hospitals' opinions about the instrument in single factor. For example, considered the performance of the product only, $70 \%$ of the hospitals believed it was very reasonable, $20 \%$ reasonable, $10 \%$ less reasonable, no hospital unreasonable. The conclusion was:

$$
\text { product performance } \rightarrow(0.7,0.2,0.1,0)
$$

In the same method they can know:

$$
\begin{aligned}
& \text { product shape } \mid \rightarrow(0.2,0.3,0.4,0.1) \\
& \text { product color } \mid \rightarrow(0.3,0.4,0.2,0.1)
\end{aligned}
$$

\subsection{Determine the Design Variables}

Through the analysis we can see that these problems can be determined the weights by comprehensive evaluation rule in fuzzy optimization method, then obtain the outcome with comprehensive evaluation.

$\mathrm{U}=$ \{product performance, product shade, product color $\}$ is the factors set;

$\mathrm{V}=\{$ very reasonable, reasonable, less reasonable, unreasonable $\}$ is the judgment set;

Judged by the four single factors, evaluation matrix R can be obtained:

$$
R=\left(\begin{array}{cccc}
0.7 & 0.2 & 0.1 & 0 \\
0.2 & 0.3 & 0.4 & 0.1 \\
0.3 & 0.4 & 0.2 & 0.1
\end{array}\right)
$$

2.2 Determine the Objective Function

$$
\begin{gathered}
B=A \circ R \\
N\left(B_{1}, \quad B\right)=1-\frac{1}{4} \sum_{\mathrm{i}=1}^{4}\left|B_{1}\left(X_{1}\right)-B\left(X_{1}\right)\right|
\end{gathered}
$$




\subsection{Determine the Constraints}

2.4 Analytical steps

the weights of hospitals' opinions: $\mathrm{A}=(0.5,0.3,0.2)$

Using fuzzy transform determined by $\mathrm{R}$ to do a comprehensive evaluation, the results of that evaluation is:

$$
\begin{aligned}
B=A \circ R & \\
& =(0.5,0.3,0.2) \circ\left(\begin{array}{cccc}
0.7 & 0.2 & 0.1 & 0 \\
0.2 & 0.3 & 0.4 & 0.1 \\
0.3 & 0.4 & 0.2 & 0.1
\end{array}\right) \\
& =(0.5,0.3,0.3,0.1)
\end{aligned}
$$

\subsection{Using C \# graphical interface, gives optimal results}

(1)Inputting the weights of factor in the appropriate input box, then click "input", if factors were not enough, click “add”; (Figure 2.5.1)

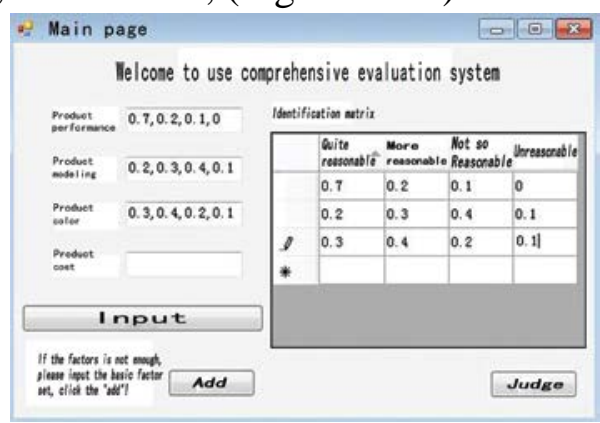

Figure 2.5.1 Factors weights

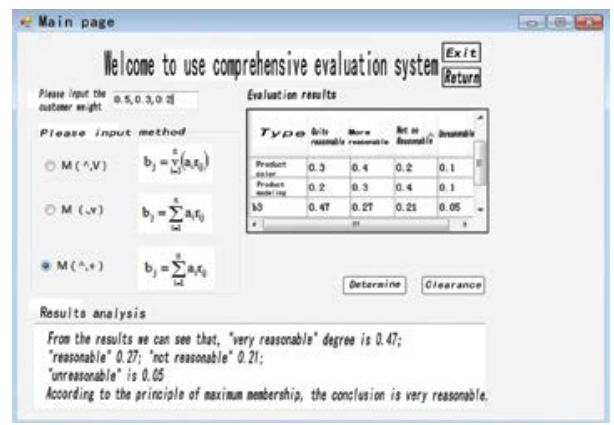

Figure 2.5.2 Results analysis

(2) In the input box, inputting the customer's weights and selecting a method arbitrarily and clicking "OK", then viewing the results of the analysis. The analysis reveals that the conclusion is very reasonable according to the principle of maximum membership. (Figure 2.5.2)

\section{Conclusions}

Fuzzy optimization design can fix the fuzzy problem better in products design. Especially the usage of C\#, which can process plenty of data quickly, has great effect on product improvement design. As for designers, this direction of design may benefit more creative thinking and make sure more time will be used in design. We've overcome lots of difficulties during the process of realization. Such as the selection and modeling of Fuzzy optimization design of actual problems, the selection of matlab, soilidworks, adams or C\# to program and solve the question and so on. Of course, this approach also has its limitations. It can not be stereotyped for the design. Sometimes a result is not the optimal will be appreciated by more people, divergent thinking will inspire a lot of creativity.

\section{References}

[1] Shi Xinmin, Hao Zhengqing. Fuzzy control and simulation. Tsinghua University press, 2008.

[2] Li Shiyong. Fuzzy control, neural control and intelligent control theory. Harbin Institute of Technology press, 1996.

[3] Huang Hongzhong. Fuzzy design. Mechanical Industry Press, 1999.

[4] Zhang Lianhong. Modern design method and its application. Tianjin University press, 2008.

[5] Zhong Zhihua, Zhou Yanwei. Modern design method. Wuhan University of Technology press, 2001.

[6] Qi Libo. C\# introduction to the classic. Tsinghua University press, 2010. 